\title{
Different prognostic implications of hepatic metastasis according to front-line treatment in non-small cell lung cancer: a real-world retrospective study
}

\author{
Myeong Geun Choi ${ }^{1}$, Chang-Min Choi ${ }^{1,2}$, Dae Ho Lee ${ }^{2}$, Sang-We Kim ${ }^{2}$, Shinkyo Yoon ${ }^{2}$, Woo Sung Kim ${ }^{1}$, \\ Wonjun $\mathrm{Ji}^{1}$, Jae Cheol Lee ${ }^{2}$ \\ ${ }^{1}$ Department of Pulmonary and Critical Care Medicine, Asan Medical Center, University of Ulsan College of Medicine, Seoul, South Korea; \\ ${ }^{2}$ Department of Oncology, Asan Medical Center, University of Ulsan College of Medicine, Seoul, South Korea \\ Contributions: (I) Conception and design: W Ji, JC Lee; (II) Administrative support: W Ji; (III) Provision of study materials or patients: CM Choi, DH \\ Lee, SW Kim, S Yoon, WS Kim, W Ji, JC Lee; (IV) Collection and assembly of data: All authors; (V) Data analysis and interpretation: MG Choi, W \\ Ji, JC Lee; (VI) Manuscript writing: All authors; (VII) Final approval of manuscript: All authors. \\ Correspondence to: Wonjun Ji, MD, PhD. Department of Pulmonary and Critical Care Medicine, Asan Medical Center, University of Ulsan College \\ of Medicine, 88, Olympic-ro 43-gil, Songpa-gu, Seoul 05505, South Korea. Email: jack1097@naver.com; Jae Cheol Lee, MD, PhD. Department \\ of Oncology, Asan Medical Center, University of Ulsan College of Medicine, 88, Olympic-ro 43-gil, Songpa-gu, Seoul 05505, South Korea. \\ Email: jclee@amc.seoul.kr.
}

Background: Although liver metastasis occurs in approximately $15 \%$ of metastatic non-small cell lung cancer (NSCLC) patients with poor prognosis, its prognostic effect in patients who receive immunotherapy is unclear. This study aimed to verify the effects of liver metastasis on the prognosis of metastatic NSCLC patients according to their first-line treatment.

Methods: Patients who were initially diagnosed with stage 4 NSCLC from January 2015 to December 2019 were analyzed in this retrospective real-world data-based study. The patients were divided into three groups according to the type of first-line chemotherapy they received: cytotoxic, targeted, and immunotherapy. Prognosis was then compared depending on the presence of liver metastasis in each treatment group.

Results: Among the 1,470 patients, 723 (49.2\%) received cytotoxic chemotherapy, 678 (46.1\%) received targeted therapy, and 69 (4.7\%) received immunotherapy as their first-line chemotherapy. A total of 234 (15.9\%) patients had liver metastasis at the initial diagnosis. The mean patient age was 63.7 years, and $59.1 \%$ were male. There was no difference in overall survival (OS) in the immunotherapy group in patients with or without liver metastasis (11.7 vs. 13.0 months, $\mathrm{P}=0.968$ ); however, patients with liver metastasis had worse outcomes in the cytotoxic and targeted therapy groups compared to patients without liver metastasis. Furthermore, in patients with liver metastasis, the immunotherapy group had a longer OS than the cytotoxic chemotherapy group (11.7 vs. 4.4 months, $\mathrm{P}<0.001)$. Liver metastasis was associated with poor outcomes (hazard ratio of 1.438), as were age, male sex, bone, adrenal gland, or soft tissue metastasis, and three or more metastatic sites; however, lymph node, brain, collateral lung, and pleura metastasis did not affect prognosis.

Conclusions: Although liver metastasis was associated with poor outcomes, it did not affect prognosis in patients who received immunotherapy.

Keywords: Liver metastasis; non-small cell lung cancer (NSCLC); immunotherapy

Submitted Mar 13, 2021. Accepted for publication May 15, 2021.

doi: $10.21037 /$ tlcr-21-206

View this article at: http://dx.doi.org/10.21037/tlcr-21-206 


\section{Introduction}

Non-small cell lung cancer (NSCLC) accounts for $85 \%$ of all cases of lung cancer, which is the leading cause of cancer deaths worldwide (1-3) despite improvements in the prognosis of metastatic lung cancer due to the development of targeted therapy and immunotherapy (4-6). The stage of lung cancer at diagnosis affects prognosis $(7,8)$, and distant metastasis is found at initial diagnosis with a frequency of approximately $40 \%$ (8-10). Additionally, prognosis varies depending on the metastatic sites. Previous studies have shown that metastasis to the liver, bone, and adrenal glands in patients with NSCLC are associated with poor outcomes $(11,12)$.

Among the various types of metastasis, liver metastasis occurs in approximately $15 \%$ of metastatic NSCLC patients with the worst prognosis (11-13). Several studies have evaluated the effect of liver metastasis on treatment response. The results of these studies have generally indicated that patients with liver metastasis had poor outcomes (14-16). However, unlike cytotoxic chemotherapy and targeted therapy, there is still debate about the effect of liver metastasis on the prognosis of patients who receive immunotherapy. Some studies have identified liver metastasis as an independent poor prognostic factor in patients who received immunotherapy $(17,18)$; conversely, the presence of liver metastasis did not affect prognosis in other studies $(19,20)$.

Until now, there has been no comprehensive analysis of outcomes or clinical characteristics according to both the type of first-line chemotherapy and the presence of liver metastasis in NSCLC patients. Therefore, this study aimed to verify the effects of liver metastasis on the prognosis of stage 4 NSCLC patients according to their first-line treatment using real-world clinical data. We present the following article in accordance with the STROBE reporting checklist (available at http://dx.doi. org/10.21037/tlcr-21-206).

\section{Methods}

\section{Study design and definition}

This study was a single-center retrospective real-world databased study. Patients who were diagnosed with NSCLC from January 2015 to December 2019 were analyzed. The patients had stage 4 cancer with distant metastasis at initial diagnosis and were over 18 years old. The patients' demographic and baseline clinical data including epidermal growth factor receptor (EGFR), anaplastic lymphoma kinase (ALK), and programmed death-ligand 1 (PD-L1) statuses at the time of diagnosis were collected. All patients received first-line systemic treatment during the analysis period. The patients were divided into three groups depending on the type of first-line chemotherapy they received: cytotoxic, targeted, and immunotherapy. The immunotherapy group included patients who received either immunotherapy alone or the combination of immunotherapy and cytotoxic chemotherapy.

The study was conducted in accordance with the Declaration of Helsinki (as revised in 2013). The study was approved by the institutional review board of Asan Medical Center (IRB No. 2020-0921) and individual consent for this retrospective analysis was waived.

\section{Selection flow of study population}

Diagnostic codes from medical records were used to extract NSCLC patients. The diagnostic codes included "adenocarcinoma", "squamous cell carcinoma", "adenosquamous cell carcinoma", "large cell carcinoma", "other non-small cell carcinoma", "unspecified lung cancer", and "malignant neoplasm of lung" from the International Classification of Disease-10 code C34. When evaluating metastatic sites, the results of the initial chest and/or abdominal pelvic computed tomography, positron emission tomography-computed tomography, or brain magnetic resonance imaging were used, and patients with the words "metastasis", "metastatic", and "metastases" in the results were extracted. The metastatic sites were then classified using these extracted results.

The exclusion criteria were patients with recurrence; patients who were stage 4 at diagnosis but had not undergone chemotherapy; patients with types of cancer other than NSCLC; or patients with other malignancies during the analysis period (Figure 1).

\section{Measurement of prognostic outcomes}

The primary outcome was a comparison of overall survival (OS) and progression-free survival (PFS) depending on the presence of liver metastasis in each treatment group, particularly in the immunotherapy group. The secondary outcomes were the distribution of metastatic sites in stage 4 NSCLC, the difference in prognosis according to metastatic sites, and other factors affecting OS and PFS in patients with stage 4 NSCLC. 


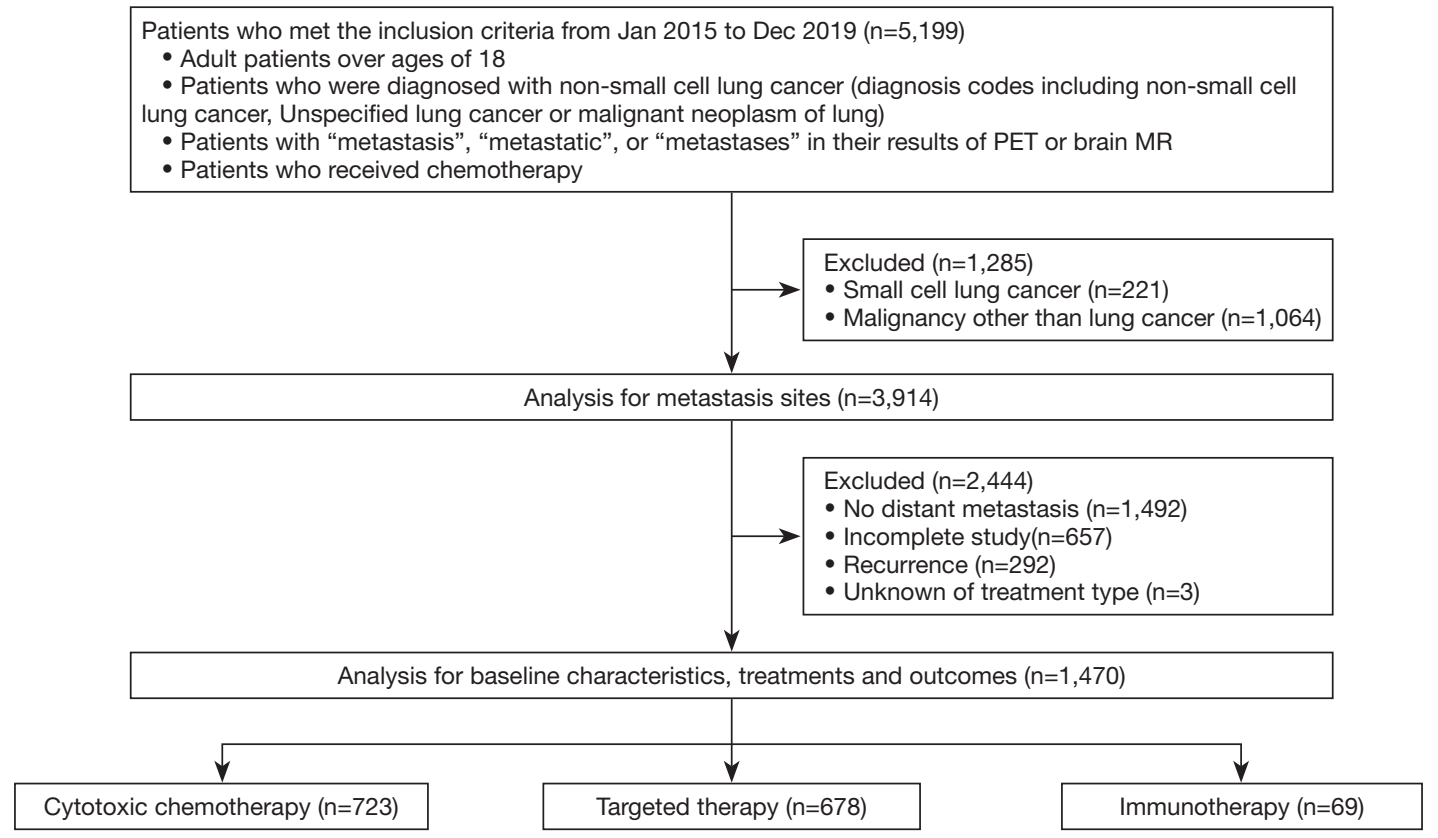

Figure 1 Consort diagram of the study.

\section{Statistical analysis}

$T$-tests were used to compare continuous variables, and Chi-square tests were used to compare categorical variables between two groups with or without liver metastasis. All values were denoted as the mean \pm standard deviation or number (percentage). Kaplan-Meier curves and the log-rank test were used to compare OS and PFS. The PFS analysis was based on the Response Evaluation Criteria in Solid Tumors guidelines (version 1.1). We used a Cox regression analysis to obtain the hazard ratio (HR) of each variable for the OS and PFS. A p-value of less than 0.05 was considered statistically significant. The statistical analyses were performed using IBM SPSS version 25.0 (IBM Corp., Armonk, NY, USA).

\section{Results}

\section{Baseline characteristics of the study population}

During the analysis period, 5,199 patients met the inclusion criteria. A total of 1,470 patients were finally analyzed after excluding 3,729 patients who met the exclusion criteria. Among them, 723 patients received cytotoxic chemotherapy, 678 received targeted therapy, and 69 received immunotherapy as their first-line chemotherapy (Figure 1 and Table 1). In particular, 30 and 39 patients in the immunotherapy group received immunotherapy alone and combination therapy, respectively. Baseline characteristics in each treatment group are presented in Table S1. Of the total population, 234 (15.9\%) patients had liver metastasis at the initial diagnosis. Bone accounted for the largest percentage of metastatic sites, followed by the pleura and extra thoracic lymph nodes. A total of $31.4 \%$ of patients had a single metastatic site and $68.6 \%$ had two or more metastatic sites (Table 2). The mean patient age was 63.7 years, and $59.1 \%$ were male. Adenocarcinoma and squamous cell carcinoma comprised $82.9 \%$ and $13.2 \%$ of the cancers, respectively. The molecular test results at diagnosis showed that $42.9 \%$ of patients had EGFR gene mutations and 6.4\% had ALK gene rearrangement (Table 1). Additionally, $55 \%$ of the patients who received first-line immunotherapy were $\mathrm{PD}-\mathrm{L} 1$ positive (PD-L1 expression $\geq 1 \%$ ) and $18.8 \%$ were PD-L1 negative (Table S2). There were no statistical differences in the baseline characteristics between the groups with and without liver metastasis.

\section{Prognostic differences according to bepatic metastasis}

The median OS of the patients with liver metastasis was 9.4 months and that of the patients without liver metastasis was 18.4 months $(\mathrm{P}<0.001)$. In the cytotoxic chemotherapy group and the targeted therapy group, the OS of patients with liver metastasis was shorter than that of patients 
Table 1 Baseline characteristics

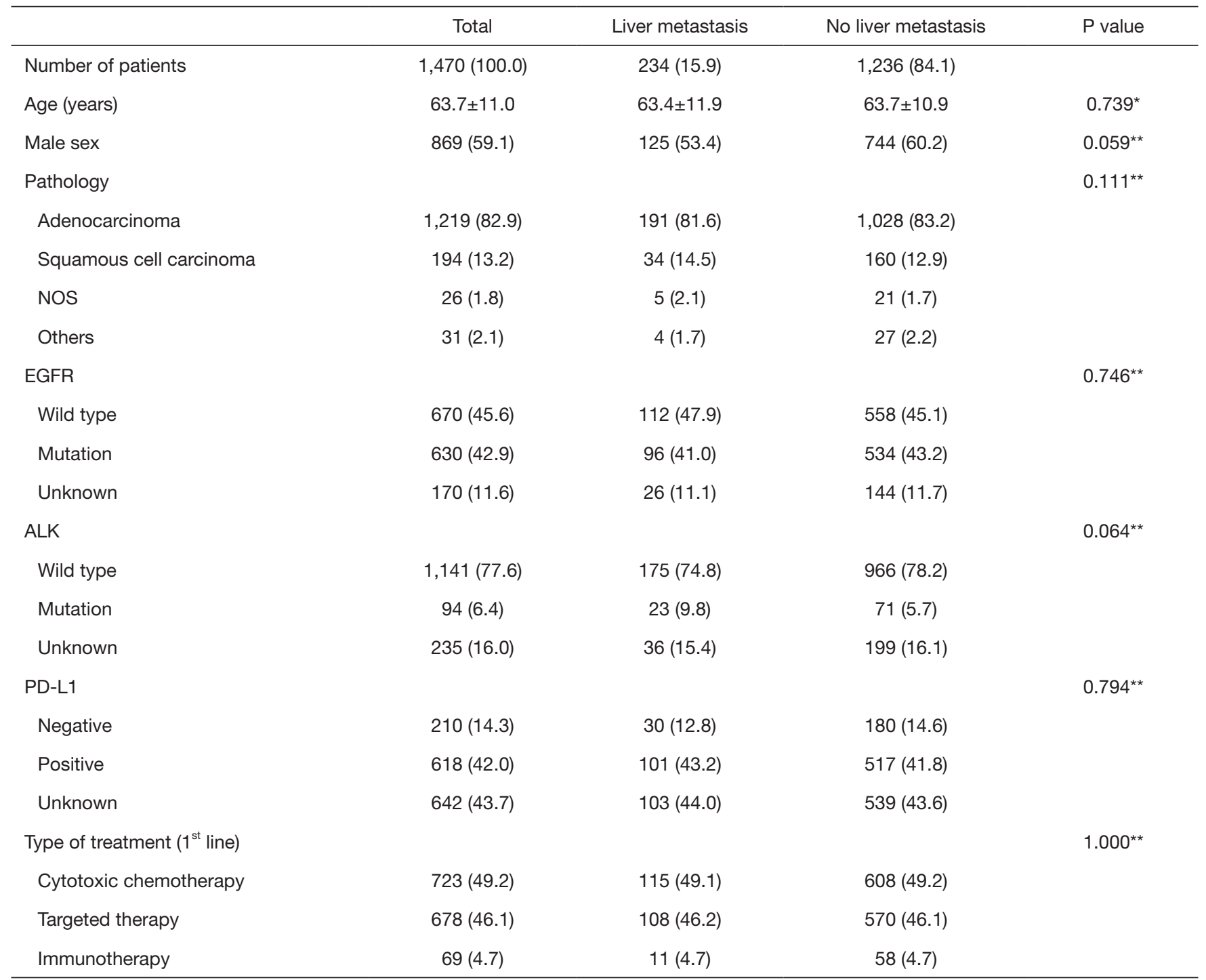

*, $t$-test; ${ }^{*}$, Chi-square test. NOS, not otherwise specified; EGFR, epidermal growth factor receptor; ALK, anaplastic lymphoma kinase; PD-L1, programmed death-ligand 1.

without liver metastasis (4.4 vs. 10.8 months, $\mathrm{P}<0.001$; 15.6 vs. 29.5 months, $\mathrm{P}<0.001$; respectively). However, there was no difference in OS depending on the presence of liver metastasis in the immunotherapy group (11.7 vs. 13.0 months, $\mathrm{P}=0.968$; Figure 2). Similar results were observed for subgroups within each group (Figure S1).

The presence or absence of liver metastasis resulted in PFS findings that were similar to the OS findings (Figure S2). The PFS of all patients without liver metastasis was longer, and even in the cytotoxic and targeted therapy groups, patients without liver metastasis had a better PFS. Similar to OS, there was no difference in PFS in the immunotherapy group in the patients with or without liver metastasis.

\section{Prognostic differences according to first-line treatment}

According to the treatment type, the median OS of the targeted therapy group was significantly longer than that of the other treatment groups $(\mathrm{P}<0.001)$. However, there was no difference in the survival curve between the cytotoxic and immunotherapy groups ( 9.7 and 13.0 months, $\mathrm{P}=0.361$ ). Conversely, in the patients with liver metastasis, the immunotherapy group had a longer OS than the cytotoxic 
Table 2 Distribution of metastatic sites

\begin{tabular}{lccc}
\hline & Total & Adenocarcinoma & Squamous cell carcinoma \\
\hline Metastatic sites & & & $34(17.5)$ \\
Liver & $234(15.9)$ & $191(15.7)$ & $56(28.9)$ \\
Lung & $471(32.0)$ & $407(33.4)$ & $77(39.7)$ \\
Extrathoracic lymph node & $580(39.5)$ & $485(39.8)$ & $75(38.7)$ \\
Pleura & $632(43.0)$ & $535(43.9)$ & $76(39.2)$ \\
Bone & $801(54.5)$ & $694(56.9)$ & $29(14.9)$ \\
Brain & $467(31.8)$ & $426(34.9)$ & $30(15.5)$ \\
Adrenal gland & $252(17.1)$ & $211(17.3)$ & $9(4.6)$ \\
Soft tissue & $100(6.8)$ & $86(7.1)$ & $31(54.4)$ \\
Number of metastatic sites & & $370(30.4)$ & $78(40.2)$ \\
One & $462(31.4)$ & $302(24.8)$ & $54(27.8)$ \\
Two & $386(26.3)$ & $547(44.9)$ & $62(32.0)$ \\
Three or more & $622(42.3)$ & & $11(19.3)$ \\
\hline
\end{tabular}
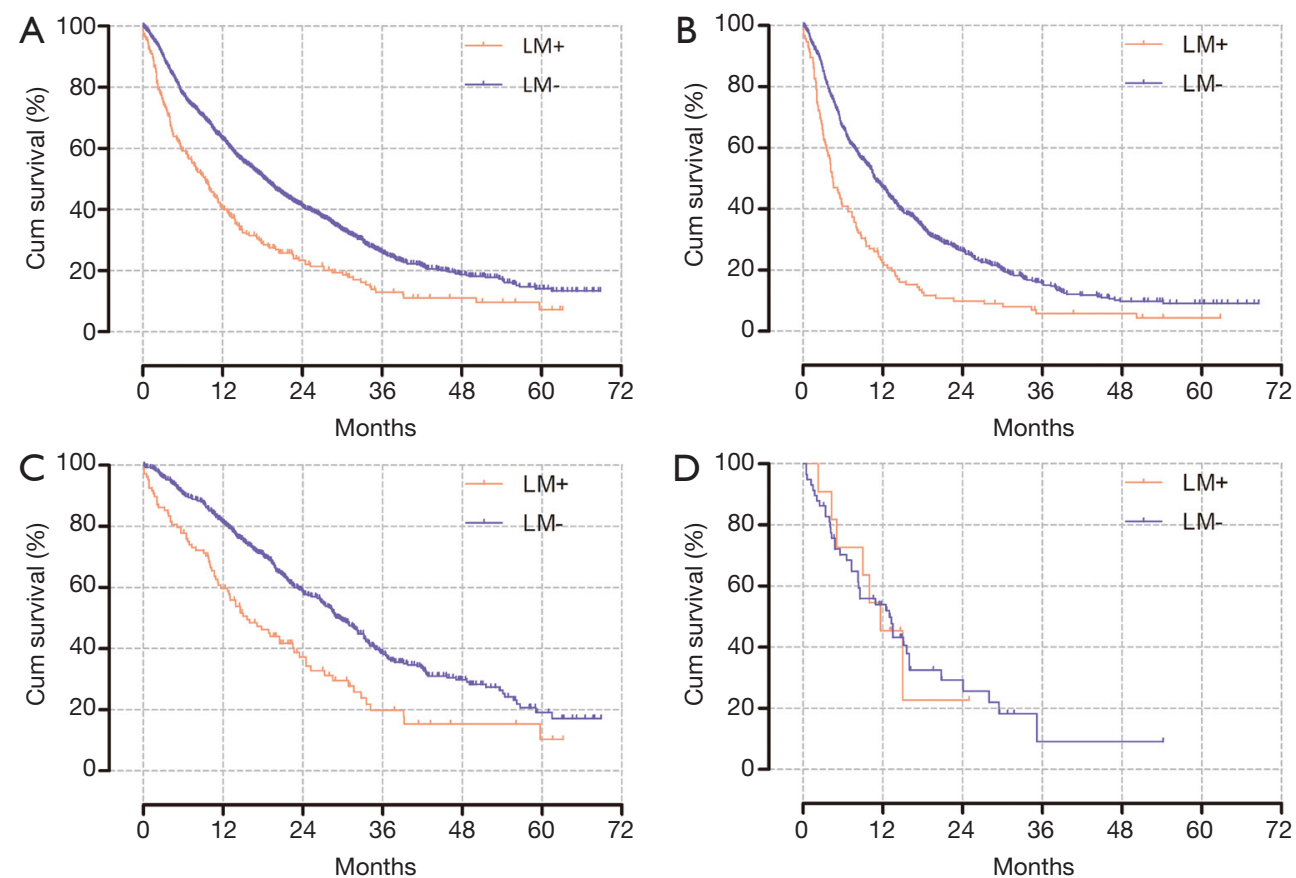

Figure 2 Kaplan-Meier curves for overall survival depending on liver metastasis. (A) Entire study population, P<0.001 [median OS: 9.4 (LM+) vs. 18.4 months (LM-)]. (B) Cytotoxic chemotherapy group, $\mathrm{P}<0.001$ [median OS: 4.4 (LM+) vs. 10.8 months (LM-)]. (C) Targeted therapy, $\mathrm{P}<0.001$ [median OS: $15.6(\mathrm{LM}+)$ vs. 29.5 months (LM-)]. (D) Immunotherapy, $\mathrm{P}=0.968$ [median OS: 11.7 (LM+) vs. 13.0 months (LM-)]. OS, overall survival; LM, liver metastasis. 

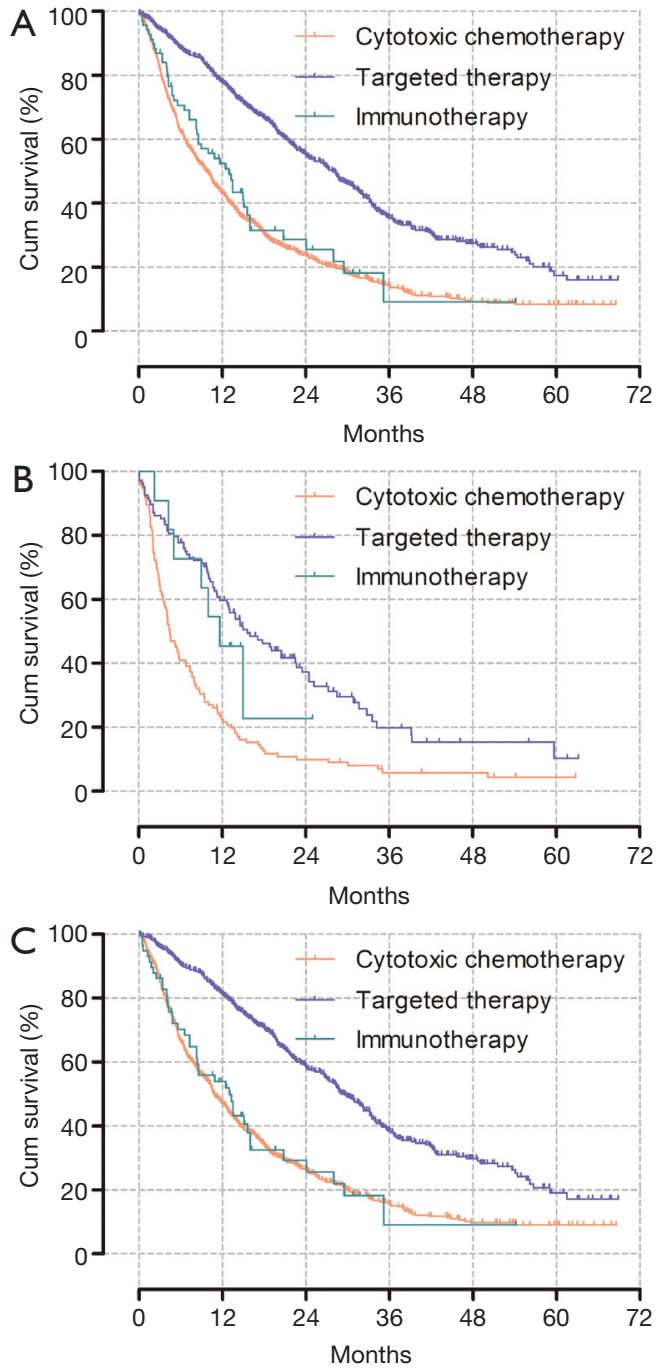

Figure 3 Kaplan-Meier curves for overall survival according to the type of first-line chemotherapy. (A) Entire population of metastatic NSCLC patients, $\mathrm{P}<0.001$ [median OS: 28.0 (target) vs. 9.7 (cytotoxic) vs. 13 (immunotherapy) months]. (B) NSCLC with liver metastasis, $\mathrm{P}<0.001$ [median OS: 15.6 (target) vs. 4.4 (cytotoxic) vs. 11.7 (immunotherapy) months]. (C) Metastatic NSCLC other than in the liver, $\mathrm{P}<0.001$ [median OS: 29.5 (target) vs. 10.8 (cytotoxic) vs. 13.0 (immunotherapy) months]. NSCLC, non-small cell lung cancer; OS, overall survival.

chemotherapy group did (11.7 vs. 4.4 months, $\mathrm{P}<0.001)$, and the survival curve between the targeted and immunotherapy groups did not differ significantly ( $\mathrm{P}=0.506$; Figure 3).

In patients with liver metastasis, the cytotoxic chemotherapy group had a significantly worse PFS than that of the targeted or immunotherapy groups, and the
PFS of the targeted therapy group was longer than that of the immunotherapy group, but this difference was not statistically significant (Figure S3).

\section{Prognostic differences according to the number and location of metastatic sites}

Although there was no difference in OS between the patients with one or two metastatic sites when considering the entire study population, patients with three or more metastatic sites had worse outcomes than patients with one or two metastatic sites $(\mathrm{P}<0.001)$. Meanwhile, there was no association between the number of metastatic sites and OS in patients with liver metastasis (Figure 4).

The prognosis in patients with metastasis occurring at sites other than the liver tended to differ from that of patients with liver metastasis. Within each treatment group, particularly within patients who received immunotherapy, bone, adrenal, or soft tissue metastasis had a significant negative effect on prognosis, unlike liver metastasis. There was no statistical difference in the prognosis of those who received immunotherapy and cytotoxic chemotherapy according to the presence or absence of these types of metastasis (Figure S4).

\section{Cox regression analysis for $O S$ and $P F S$}

In the multivariate Cox regression analysis for OS, the HR of age was 1.024 and the HR of male sex was 1.243. Furthermore, the HR for EGFR mutations was 0.457 and the HR for ALK rearrangement was 0.352 , with good prognosis. Along with liver metastasis ( $H R=1.438)$, bone, adrenal gland, and soft tissue metastasis were significantly associated with poor outcomes. Conversely, lymph node, brain, collateral lung, and pleura metastasis did not affect prognosis. Although two metastatic sites did not statistically affect OS when compared with a single metastatic site, three or more metastatic sites were associated with poor outcomes ( $\mathrm{HR}=1.466$; Table 3). Similar results were found in terms of PFS, in which age, male sex, and squamous cell carcinoma were associated with poor outcomes. No EGFR mutation, no ALK rearrangement, three or more metastatic sites, and metastasis to the liver, bone, and adrenal glands were also associated with a poor PFS (Table S3).

\section{Discussion}

This is the first study that used large real-world data to 

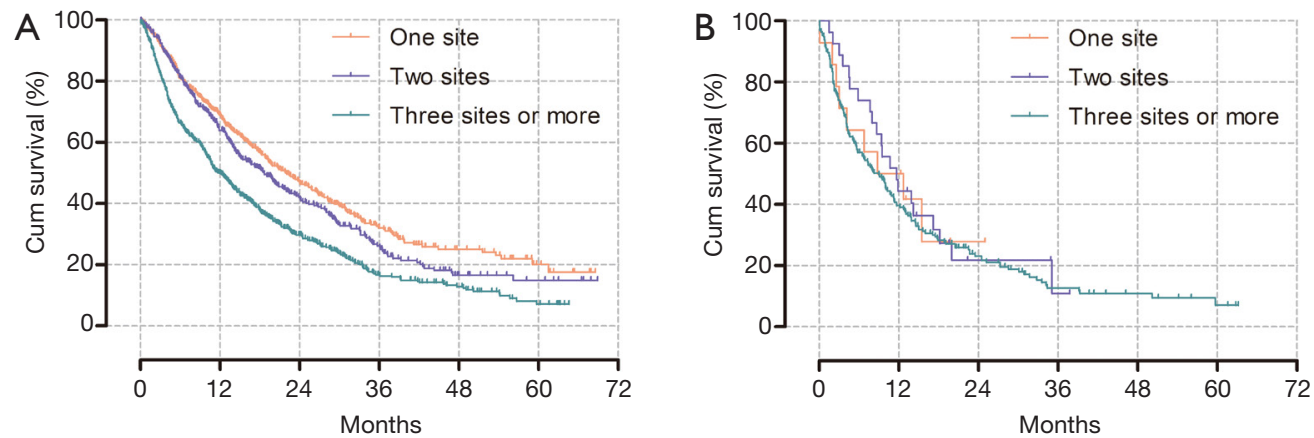

Figure 4 Kaplan-Meier curves for overall survival according to the number of metastatic sites. (A) Entire study population, $\mathrm{P}<0.001$ (median OS: 22.1 vs. 18.7 vs. 12.0 months; 1 vs. 2 sites, $\mathrm{P}=0.071 ; 1$ or 2 vs. 3 or more sites, $\mathrm{P}<0.001$ ). (B) Patients with liver metastasis, $\mathrm{P}=0.681$ (median OS: 8.8 vs. 11.7 vs. 9 months). OS, overall survival.

Table 3 Cox regression analysis for overall survival

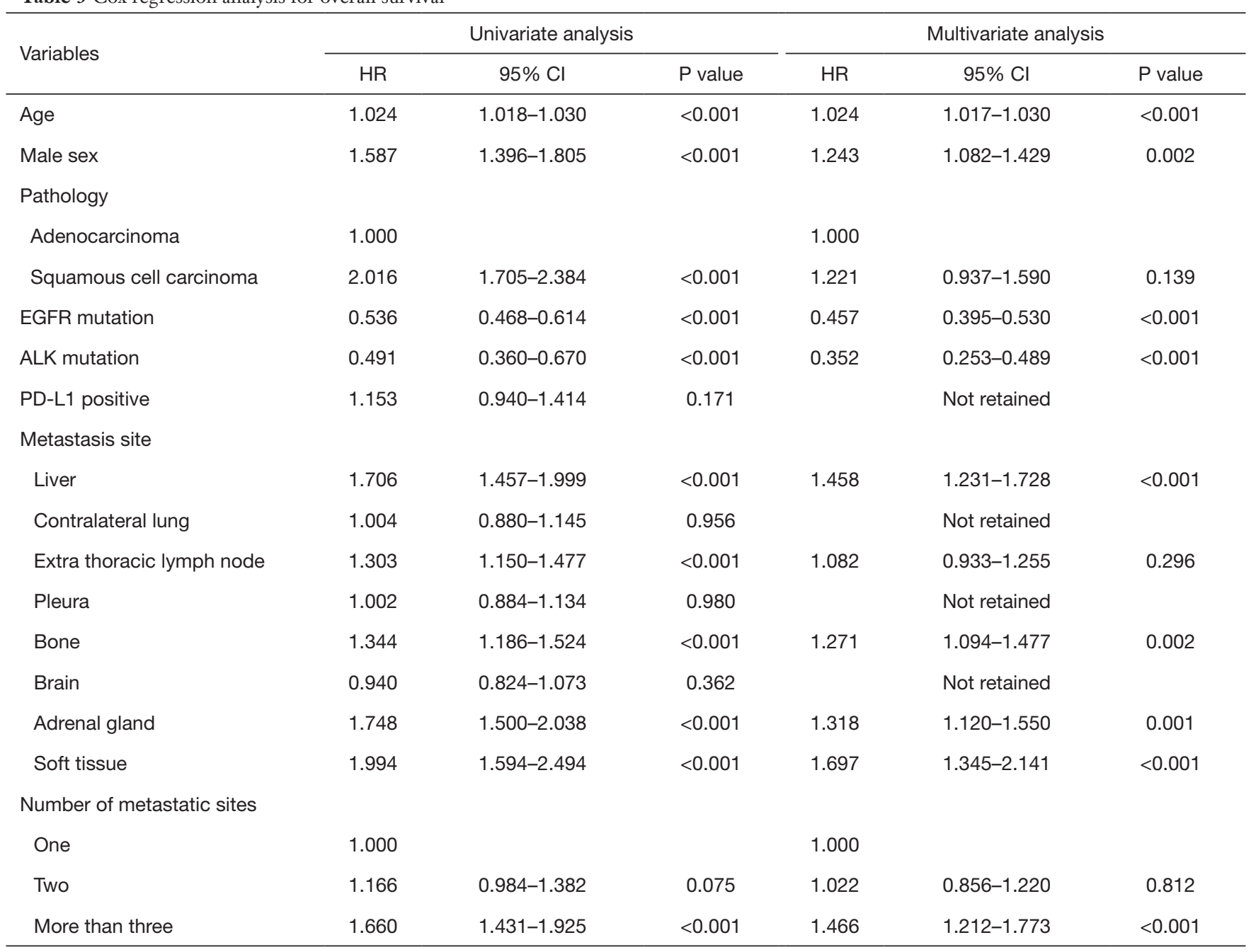

EGFR, epidermal growth factor receptor; ALK, anaplastic lymphoma kinase; PD-L1, programmed death-ligand 1. 
perform a comprehensive, multidimensional analysis of survival outcomes according to the presence of liver metastasis in each treatment group. NSCLC patients with liver metastasis generally had a worse prognosis than patients without liver metastasis did. However, in the immunotherapy group, liver metastasis did not significantly affect survival. While there is no doubt regarding the use of tyrosine kinase inhibitor (TKI) or immune checkpoint inhibitor (ICI) with/without cytotoxic chemotherapy as first-line treatment depending on the presence of driver mutation in this era, we could emphasize the need for certain mechanisms to be revealed wherein liver metastasis does not affect prognosis in patients who have undergone immunotherapy, which could serve as another basis for developing treatment strategies for patients with liver metastasis.

Prior to the use of targeted therapy, liver metastasis had been identified as a factor associated with poor outcomes in NSCLC patients who received cytotoxic chemotherapy (21). The findings are not much different for patients receiving targeted therapy such as TKI. Some studies have reported significantly shorter PFS and OS in NSCLC patients with liver metastasis who receive TKIs as the first-line treatment after confirming the presence of EGFR mutations $(14,16)$. The results of these studies support our findings.

However, in this present study, there was no difference in OS in patients with or without liver metastasis in the immunotherapy group. Thus far, the effect of liver metastasis on the response to immunotherapy remains controversial. Some studies have shown that the response to immunotherapy is poor if liver metastasis is present in patients receiving immunotherapy $(22,23)$. These results are thought to be due to the relationship between the liver and immune tolerance. Since the liver is responsible for immunity, liver metastasis can reduce the activation of $\mathrm{CD}^{+} \mathrm{T}$-cells and increase the immunologic tolerance against cancer $(15,22)$. However, some studies have shown opposite results. Recently, a meta-analysis showed that there was no difference in outcome depending on liver metastasis in patients who received both immunotherapy and conventional chemotherapy (24). Furthermore, liver metastasis was not an independent prognostic factor for OS or PFS in a multivariate analysis of patients who received ICIs in previous studies $(19,20)$.

Several reasons can be inferred regarding why liver metastasis does not affect the prognosis of patients who receive immunotherapy. In one meta-analysis involving five clinical trials, ICIs significantly improved the OS of patients with liver metastasis (25). Another previous study showed that the anti-angiogenesis effect of conventional chemotherapy combined with immunotherapy increases the response of immunotherapy by blocking vascular endothelial growth factor (VEGF)-related immunosuppression and augmenting T-cell activation (26). In this current study, in patients with liver metastasis, the outcome of immunotherapy was better than that of conventional chemotherapy. Although there were few patients who received VEGF inhibitors, as previous studies inferred, it is assumed that some benefits of immunotherapy with/without chemotherapy in patients with liver metastasis reduce the negative effect of liver metastasis on the response to immunotherapy. Further large-scale studies are required to reveal the mechanism responsible for this finding.

In addition to liver metastasis, soft tissue, adrenal gland, and bone metastases have been confirmed as poor prognostic factors. These findings are similar to the results of previous studies $(11,12,27)$. Although the reason is not yet clear, the HR for the OS of soft tissue metastasis was the highest among all metastatic sites. If cancer cells spread to uncommon sites such as soft tissue, which is usually a difficult site for cancer cells to survive, such cells are likely aggressive and might have already spread to other various organs (27). Interestingly, some metastatic sites including the brain and extra thoracic lymph nodes did not affect prognosis in the multivariate analysis. This might have been caused by the ease of local treatment, such as radiotherapy, on these sites compared with other metastatic sites. Patients with oligo metastatic NSCLC who received local consolidative therapy had significantly better outcomes $(28,29)$. For these metastatic sites, we can consider aggressive local treatment in addition to systemic treatment.

There are several limitations in this study. First, this study was a single-center retrospective study. However, the Asan Medical Center is the largest tertiary-care hospital in Korea, and this current study is meaningful enough because real-world data from this large center have been used to analyze prognosis according to the first-line treatment and metastatic sites. Second, since the data were collected from 2015, the effect of immunotherapy as a first-line treatment, which has been proven by recent randomized controlled trials (30-32), has not yet been reflected. Moreover, immunotherapy with/without chemotherapy as first-line treatment is currently approved in Korea, but some patients refuse immunotherapy as first-line treatment due to financial burden as insurance does not cover it. We believe that this may be the reason for the low number of 
patients $(n=69)$ who underwent immunotherapy as their first-line treatment, which includes only 11 patients with liver metastasis. There might also be different selections of immunotherapy with/without chemotherapy according to PD-L1 status or tumor mutational burden in the analysis period; hence, there may have been no difference in prognosis between the patients who received cytotoxic chemotherapy and immunotherapy in the present study. Nevertheless, it is notable that liver metastasis has different effects on prognosis in patients undergoing immunotherapy and those undergoing other treatments.

Third, the immunotherapy regime was also heterogeneous and patients with immunotherapy alone and combination therapy were analyzed together. Although, subgroup analysis showed results similar to those of the total immunotherapy group, further study is warranted to compare these subgroups with liver metastasis. In addition, outcomes were compared according to only the type of first-line chemotherapy. The treatment after firstline therapy may have affected the outcome; thus, further analysis according to subsequent treatment is necessary. Tumor burden or performance status, which could affect prognosis, should also be considered in future studies.

\section{Conclusions}

In conclusion, liver metastasis was associated with poor outcomes. Patients who received cytotoxic or targeted therapy had a poor prognosis if liver metastasis was present. However, liver metastasis did not affect prognosis in patients who received immunotherapy.

\section{Acknowledgments}

The authors would like to thank Enago (http://www.enago. co.kr) for the English language review.

Funding: This work was supported by Asan Institute for Life Sciences and Corporate Relations of Asan Medical Center, Seoul, Korea (grant number 2019IL0840 to W. Ji).

\section{Footnote}

Reporting Checklist: The authors have completed the STROBE reporting checklist. Available at http://dx.doi. org/10.21037/tlcr-21-206

Data Sharing Statement: Available at http://dx.doi. org/10.21037/tlcr-21-206
Peer Review File: Available at http://dx.doi.org/10.21037/ tlcr-21-206

Conflict of Interest: All authors have completed the ICMJE uniform disclosure form (available at http://dx.doi. org/10.21037/tlcr-21-206). The authors have no conflicts of interest to declare.

Etbical Statement: The authors are accountable for all aspects of the work in ensuring that questions related to the accuracy or integrity of any part of the work are appropriately investigated and resolved. The study was conducted in accordance with the Declaration of Helsinki (as revised in 2013). The study was approved by the institutional review board of Asan Medical Center (IRB No. 2020-0921) and individual consent for this retrospective analysis was waived.

Open Access Statement: This is an Open Access article distributed in accordance with the Creative Commons Attribution-NonCommercial-NoDerivs 4.0 International License (CC BY-NC-ND 4.0), which permits the noncommercial replication and distribution of the article with the strict proviso that no changes or edits are made and the original work is properly cited (including links to both the formal publication through the relevant DOI and the license). See: https://creativecommons.org/licenses/by-nc-nd/4.0/.

\section{References}

1. Siegel RL, Miller KD, Jemal A. Cancer statistics, 2020. CA Cancer J Clin 2020;70:7-30.

2. Jung KW, Won YJ, Kong HJ, et al. Cancer statistics in Korea: incidence, mortality, survival, and prevalence in 2015. Cancer Res Treat 2018;50:303-16.

3. Dela Cruz CS, Tanoue LT, Matthay RA. Lung cancer: epidemiology, etiology, and prevention. Clin Chest Med 2011;32:605-44.

4. Maemondo M, Inoue A, Kobayashi K, et al. Gefitinib or chemotherapy for non-small-cell lung cancer with mutated EGFR. N Engl J Med 2010;362:2380-8.

5. Solomon BJ, Mok T, Kim DW, et al. First-line crizotinib versus chemotherapy in ALK-positive lung cancer. N Engl J Med 2014;371:2167-77.

6. Reck M, Rodriguez-Abreu D, Robinson AG, et al. Pembrolizumab versus chemotherapy for PD-L1positive non-small-cell lung cancer. N Engl J Med 2016;375:1823-33. 
7. Cetin K, Ettinger DS, Hei YJ, et al. Survival by histologic subtype in stage IV nonsmall cell lung cancer based on data from the surveillance, epidemiology and end results program. Clin Epidemiol 2011;3:139-48.

8. Choi CM, Kim HC, Jung CY, et al. Report of the Korean association of lung cancer registry (KALC-R), 2014. Cancer Res Treat 2019;51:1400-10.

9. Little AG, Gay EG, Gaspar LE, et al. National survey of non-small cell lung cancer in the United States: epidemiology, pathology and patterns of care. Lung Cancer 2007;57:253-60.

10. Morgensztern D, Ng SH, Gao F, et al. Trends in stage distribution for patients with non-small cell lung cancer: a national cancer database survey. J Thorac Oncol 2010;5:29-33.

11. Tamura T, Kurishima K, Nakazawa K, et al. Specific organ metastases and survival in metastatic non-small-cell lung cancer. Mol Clin Oncol 2015;3:217-21.

12. Riihimäki M, Hemminki A, Fallah M, et al. Metastatic sites and survival in lung cancer. Lung Cancer 2014;86:78-84.

13. Ren Y, Dai C, Zheng H, et al. Prognostic effect of liver metastasis in lung cancer patients with distant metastasis. Oncotarget 2016;7:53245-53.

14. Jiang T, Cheng R, Zhang G, et al. Characterization of Liver Metastasis and Its Effect on Targeted Therapy in EGFR-mutant NSCLC: A Multicenter Study. Clin Lung Cancer 2017;18:631-9.e2.

15. Sridhar S, Paz-Ares L, Liu H, et al. Prognostic significance of liver metastasis in durvalumab-treated lung cancer patients. Clin Lung Cancer 2019;20:e601-8.

16. Wu KL, Tsai MJ, Yang CJ, et al. Liver metastasis predicts poorer prognosis in stage IV lung adenocarcinoma patients receiving first-line gefitinib. Lung Cancer 2015;88:187-94.

17. Morita R, Okishio K, Shimizu J, et al. Real-world effectiveness and safety of nivolumab in patients with non-small cell lung cancer: a multicenter retrospective observational study in Japan. Lung Cancer 2020;140:8-18.

18. Yang L, Hao X, Hu X, et al. Superior efficacy of immunotherapy-based combinations over monotherapy for EGFR-mutant non-small cell lung cancer acquired resistance to EGFR-TKIs. Thorac Cancer 2020;11:3501-9.

19. Qiao M, Zhou F, Hou L, et al. Efficacy of immunecheckpoint inhibitors in advanced non-small cell lung cancer patients with different metastases. Ann Transl Med 2021;9:34.

20. Shiroyama T, Suzuki H, Tamiya M, et al. Clinical characteristics of liver metastasis in nivolumab-treated patients with non-small cell lung cancer. Anticancer Res 2018;38:4723-9.

21. Hoang T, Xu R, Schiller JH, et al. Clinical model to predict survival in chemonaive patients with advanced non-small-cell lung cancer treated with third-generation chemotherapy regimens based on eastern cooperative oncology group data. J Clin Oncol 2005;23:175-83.

22. Tumeh PC, Hellmann MD, Hamid O, et al. Liver metastasis and treatment outcome with anti-PD-1 monoclonal antibody in patients with melanoma and NSCLC. Cancer Immunol Res 2017;5:417-24.

23. Topalian SL, Hodi FS, Brahmer JR, et al. Five-year survival and correlates among patients with advanced melanoma, renal cell carcinoma, or non-small cell lung cancer treated with nivolumab. JAMA Oncol 2019;5:1411-20.

24. Qin BD, Jiao XD, Liu J, et al. The effect of liver metastasis on efficacy of immunotherapy plus chemotherapy in advanced lung cancer. Crit Rev Oncol Hematol 2020;147:102893.

25. Yang K, Li J, Bai C, et al. Efficacy of immune checkpoint inhibitors in non-small-cell lung cancer patients with different metastatic sites: a systematic review and metaanalysis. Front Oncol 2020;10:1098.

26. Wallin JJ, Bendell JC, Funke R, et al. Atezolizumab in combination with bevacizumab enhances antigen-specific T-cell migration in metastatic renal cell carcinoma. Nat Commun 2016;7:12624.

27. Niu FY, Zhou Q, Yang JJ, et al. Distribution and prognosis of uncommon metastases from non-small cell lung cancer. BMC Cancer 2016;16:149.

28. Gomez DR, Tang C, Zhang J, et al. Local consolidative therapy vs. maintenance therapy or observation for patients with oligometastatic non-small-cell lung cancer: long-term results of a multi-institutional, phase II, randomized study. J Clin Oncol 2019;37:1558-65.

29. Gomez DR, Blumenschein GR, Jr., Lee JJ, et al. Local consolidative therapy versus maintenance therapy or observation for patients with oligometastatic non-smallcell lung cancer without progression after first-line systemic therapy: a multicentre, randomised, controlled, phase 2 study. Lancet Oncol 2016;17:1672-82.

30. Mok TSK, Wu YL, Kudaba I, et al. Pembrolizumab versus chemotherapy for previously untreated, PD-L1expressing, locally advanced or metastatic non-small-cell lung cancer (KEYNOTE-042): a randomised, open-label, controlled, phase 3 trial. Lancet 2019;393:1819-30.

31. Langer CJ, Gadgeel SM, Borghaei H, et al. Carboplatin 
and pemetrexed with or without pembrolizumab for advanced, non-squamous non-small-cell lung cancer: a randomised, phase 2 cohort of the open-label

KEYNOTE-021 study. Lancet Oncol 2016;17:1497-508.
32. Hellmann MD, Ciuleanu TE, Pluzanski A, et al. Nivolumab plus Ipilimumab in lung cancer with a high tumor mutational burden. $\mathrm{N}$ Engl J Med 2018;378:2093-104.

Cite this article as: Choi MG, Choi CM, Lee DH, Kim SW, Yoon S, Kim WS, Ji W, Lee JC. Different prognostic implications of hepatic metastasis according to frontline treatment in non-small cell lung cancer: a real-world retrospective study. Transl Lung Cancer Res 2021;10(6):25512561. doi: 10.21037/tlcr-21-206 


\section{Supplementary}

Table S1 Comparison of baseline characteristics among treatment groups

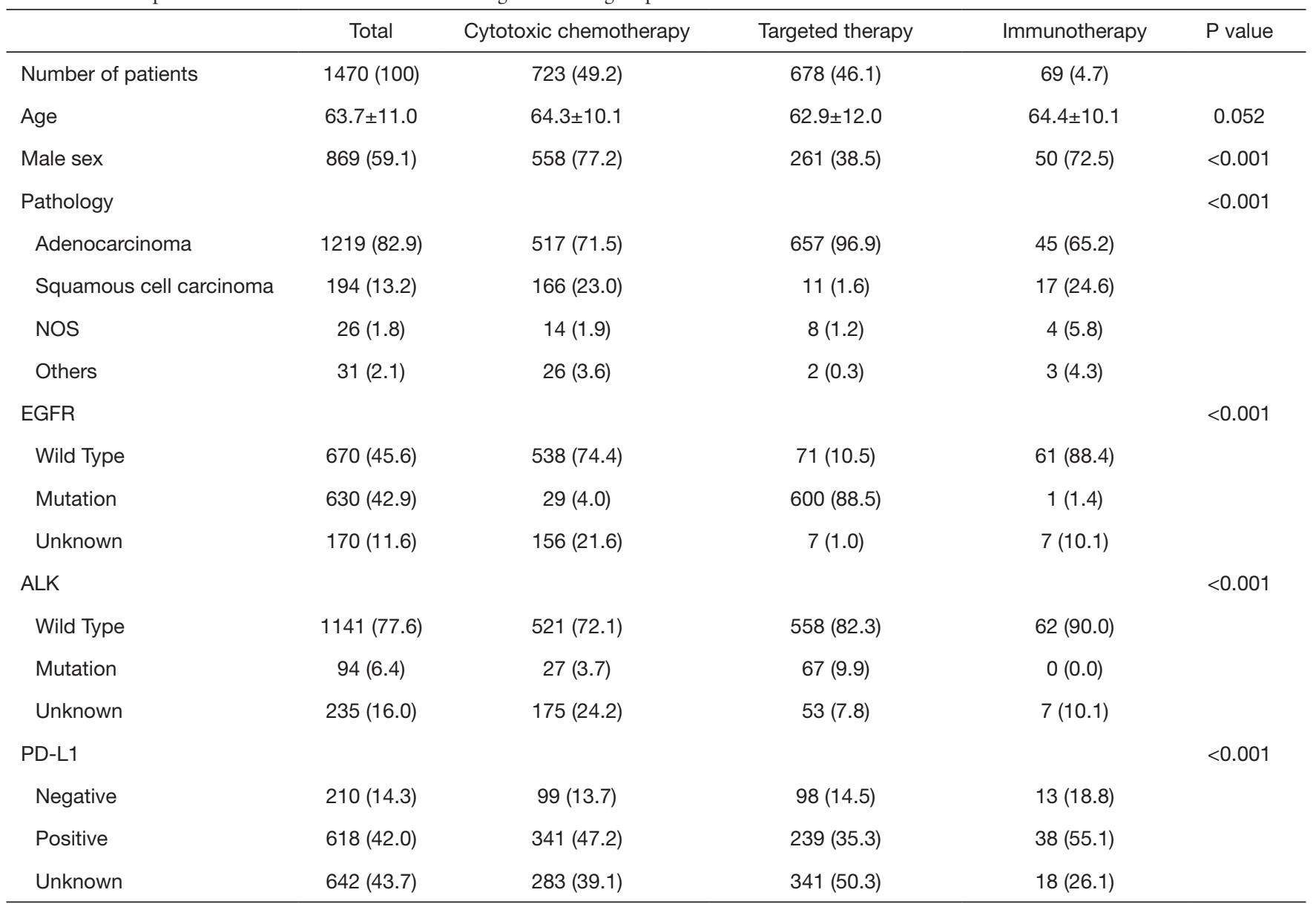

NOS, not otherwise specified; EGFR, epidermal growth factor receptor; ALK, anaplastic lymphoma kinase; PD-L1, programmed deathligand.

Table S2 PD-L1 status in the immunotherapy group

\begin{tabular}{lccc}
\hline & Total $(\mathrm{n}=69)$ & Liver metastasis $(\mathrm{n}=11)$ & No liver metastasis $(\mathrm{n}=58)$ \\
\hline$\geq 50 \%$ & $27(39.1)$ & $5(45.5)$ & $22(37.9)$ \\
$1-49 \%$ & $11(15.9)$ & $2(18.2)$ & $9(15.5)$ \\
$<1 \%$ & $13(18.8)$ & $2(18.2)$ & $11(19.0)$ \\
Unknown & $18(26.1)$ & $2(18.2)$ & $16(27.6)$ \\
\hline
\end{tabular}

PD-L1, programmed death-ligand 1. 
A

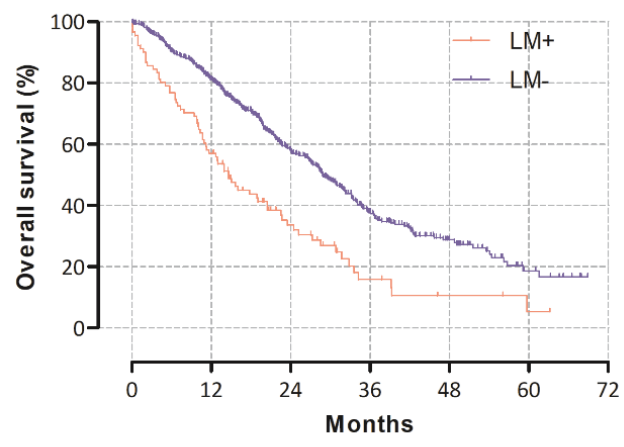

C

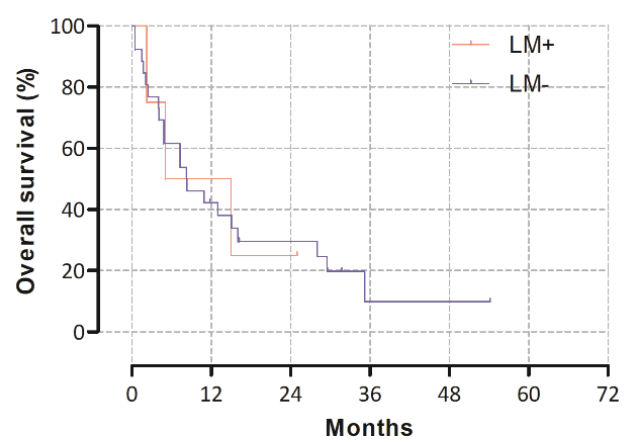

B

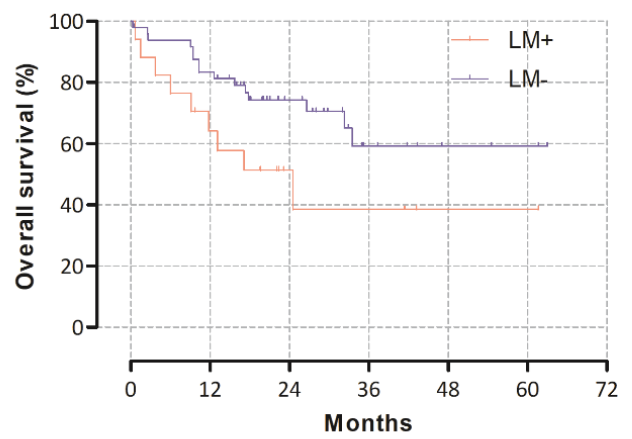

D

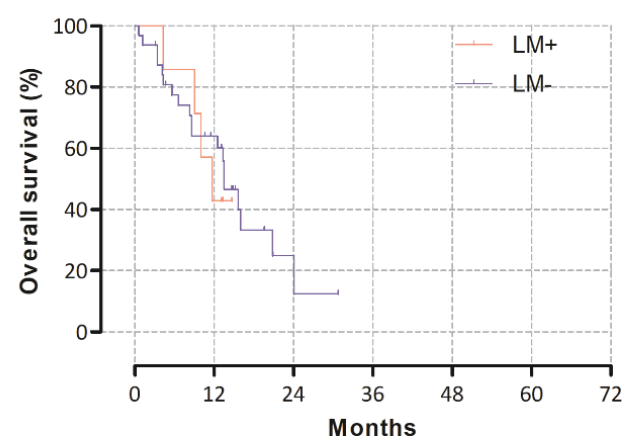

Figure S1 Subgroup analysis for overall survival with respect to liver metastasis. (A) EGFR-TKI subgroup in the targeted therapy group, $\mathrm{P}<0.001$ [median OS: $14.6(\mathrm{LM}+)$ vs. 28.8 months (LM-)]. (B) ALK-TKI subgroup in the targeted therapy group, $\mathrm{P}=0.041$ [median OS: 24.5 $(\mathrm{LM}+)$ vs. N/E months (LM-)]. (C) Immunotherapy alone subgroup in the immunotherapy group, P=0.944 [median OS: 10.1 (LM+) vs. 8.4 months (LM-)]. (D) Combination therapy subgroup in the immunotherapy group, $\mathrm{P}=0.772$ [median OS: 11.7 (LM+) vs. 13.5 months (LM-)]. EGFR, epidermal growth factor receptor; TKI, tyrosine kinase inhibitors; OS, overall survival; LM, liver metastasis; ALK, anaplastic lymphoma kinase. 
A

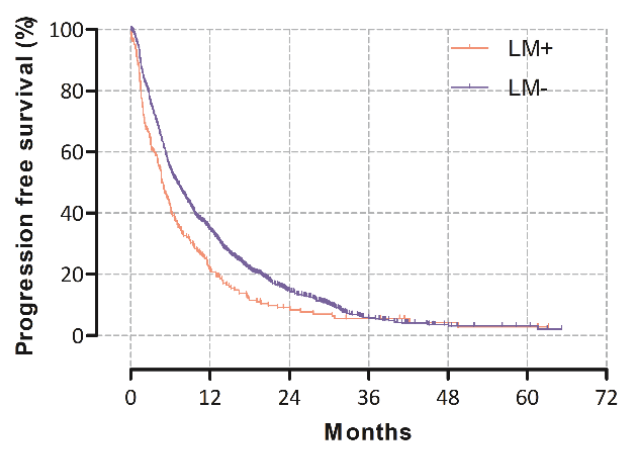

C

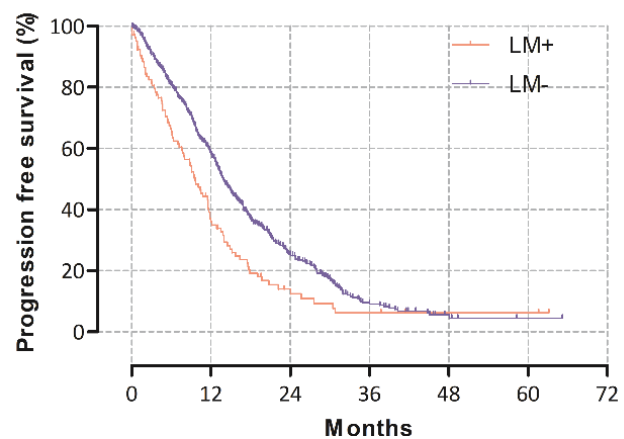

B

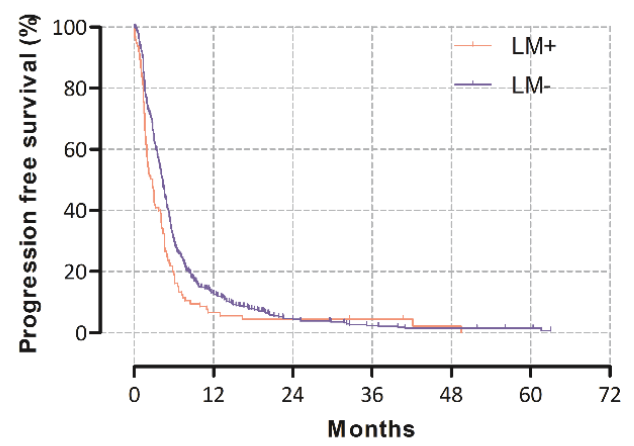

D

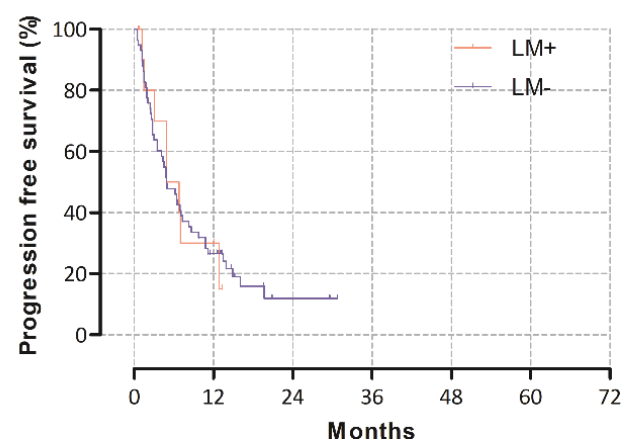

Figure S2 Progression-free survival according to liver metastasis. (A) Entire study population ( $\mathrm{P}<0.001$, median PFS: 4.7 (LM+) vs. 7.0 months (LM-)]. (B) Cytotoxic chemotherapy ( $\mathrm{P}=0.002$, median PFS: $2.8(\mathrm{LM}+)$ vs. 4.3 months (LM-)]. (C) Targeted therapy $(\mathrm{P}<0.001$, median PFS: 9.6 (LM+) vs. 13.8 months (LM-)]. (D) Immunotherapy ( $\mathrm{P}=0.955$, median PFS: 4.9 (LM+) vs. 4.9 months (LM-)]. PFS, progression-free survival; LM, liver metastasis. 

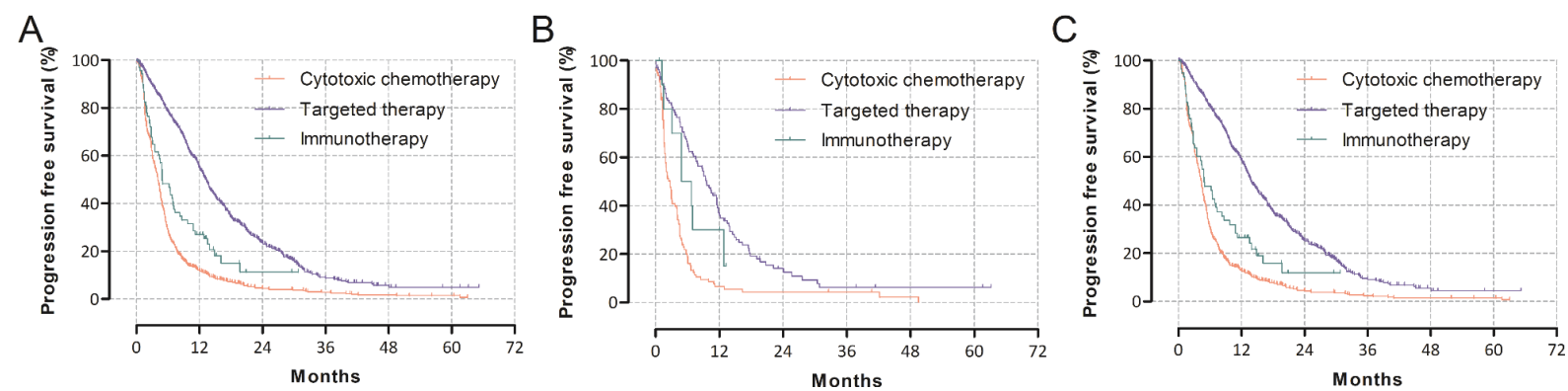

Figure S3 Progression-free survival according to treatment. (A) Entire population of metastatic NSCLC patients [ $[\mathrm{P}<0.001$, median PFS: 4.1 (cytotoxic) vs. 13.3 (target) vs. 4.9 (IO) months]. (B) NSCLC with liver metastasis [P<0.001, median PFS: 2.8 (cytotoxic) vs. 9.6 (target) vs. 4.9 (IO) months]. (C) Metastatic NSCLC other than in the liver [P<0.001, median PFS: 4.3 (cytotoxic) vs. 13.8 (target) vs. 4.9 (IO) months]. NSCLC, non-small cell lung cancer; PFS, progression-free survival; IO, immunotherapy.
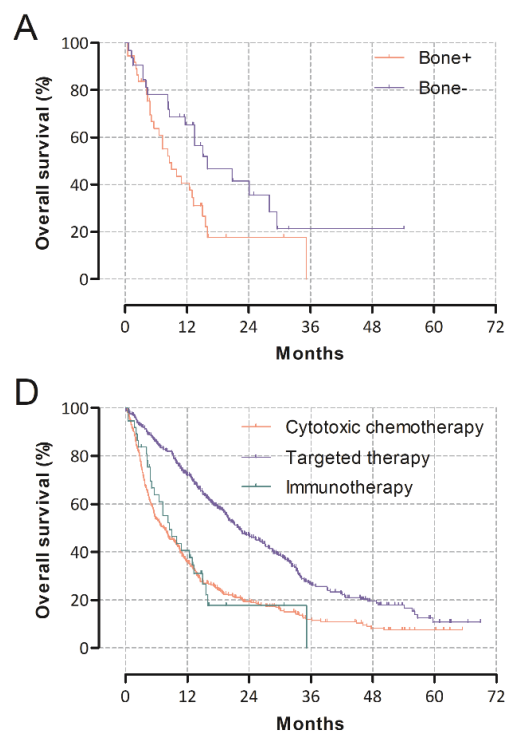

$\mathrm{B}$
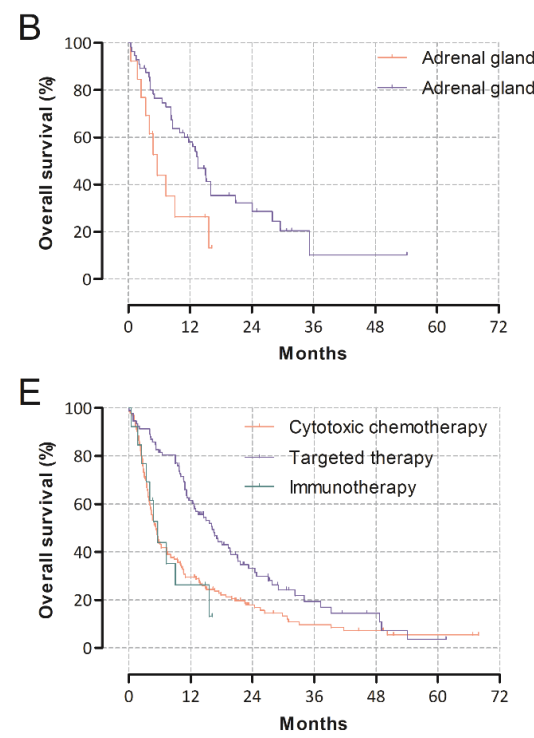
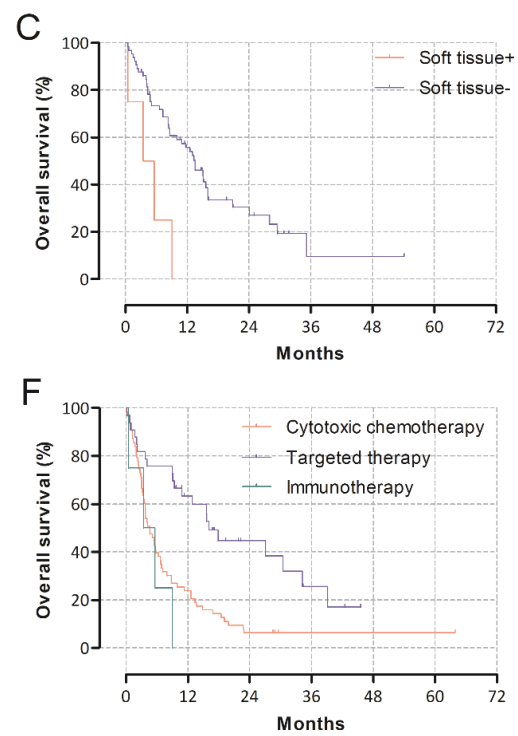

Figure S4 Overall survival with metastasis sites other than the liver. (A,B,C) Overall survival in patients who received immunotherapy with respect to the presence of metastasis (A. bone, $\mathrm{P}=0.033$; B. adrenal gland, $\mathrm{P}=0.035$; C. soft tissue, $\mathrm{P}=0.005)$. (D,E,F) Overall survival with respect to first-line chemotherapy (D. bone, $\mathrm{P}<0.001$; E. adrenal gland, $\mathrm{P}<0.001$; F. soft tissue metastasis, $\mathrm{P}<0.001$ ). 
Table S3 Cox regression analysis for progression-free survival

\begin{tabular}{|c|c|c|c|c|c|c|}
\hline Variables & \multicolumn{3}{|c|}{ Univariate analysis } & \multicolumn{3}{|c|}{ Multivariate analysis } \\
\hline Age & 1.008 & $1.003-1.013$ & 0.003 & 1.006 & $1.000-0.011$ & 0.049 \\
\hline Male sex & 1.531 & $1.363-1.719$ & $<0.001$ & 1.155 & $1.018-1.311$ & 0.026 \\
\hline \multicolumn{7}{|l|}{ Pathology } \\
\hline Squamous cell carcinoma & 2.270 & $1.931-2.669$ & $<0.001$ & 1.619 & $1.261-2.078$ & $<0.001$ \\
\hline EGFR mutation & 0.474 & $0.419-0.536$ & $<0.001$ & 0.420 & $0.366-0.482$ & $<0.001$ \\
\hline ALK mutation & 0.733 & $0.572-0.939$ & 0.014 & 0.424 & $0.323-0.557$ & $<0.001$ \\
\hline PD-L1 positive & 1.147 & $0.966-1.361$ & 0.119 & & Not retained & \\
\hline Contralateral lung & 0.985 & $0.873-1.111$ & 0.804 & & Not retained & \\
\hline Extrathoracic lymph node & 1.325 & $1.180-1.488$ & $<0.001$ & 1.086 & $0.945-1.248$ & 0.245 \\
\hline Pleura & 0.962 & $0.859-1.078$ & 0.507 & & Not retained & \\
\hline Bone & 1.177 & $1.051-1.319$ & 0.005 & 1.198 & $1.049-1.368$ & 0.008 \\
\hline Brain & 0.850 & $0.752-0.960$ & 0.009 & 0.966 & $0.837-1.115$ & 0.637 \\
\hline Adrenal gland & 1.707 & $1.475-1.976$ & $<0.001$ & 1.462 & $1.250-1.710$ & $<0.001$ \\
\hline Soft tissue & 1.542 & $1.241-1.916$ & $<0.001$ & 1.172 & $0.932-1.473$ & 0.174 \\
\hline \multicolumn{7}{|l|}{ Number of metastatic sites } \\
\hline
\end{tabular}

EGFR, epidermal growth factor receptor; ALK, anaplastic lymphoma kinase; PD-L1, programmed death-ligand 1; Cl, confidence interval; $\mathrm{HR}$, hazard ratio. 